\title{
Talaromycosis-Associated Secondary Hemophagocytic Lymphohistiocytosis in Nine Human Immunodeficiency Virus-Negative Patients: A Multicenter Retrospective Study
}

This article was published in the following Dove Press journal:

Infection and Drug Resistance

Mianluan Pan

Ye Qiu

Wen Zeng

Shudan Tang

Xin Feng

Jingmin Deng

Xuan Wei

Zhiyi He

Jianquan Zhang

Department of Respiratory and Critical Care Medicine, The First Affiliated Hospital of Guangxi Medical University, Nanning, People's Republic of China
Correspondence: Jianquan Zhang Department of Respiratory and Critical Care Medicine, The First Affiliated Hospital of Guangxi Medical University, Nanning 53002I, Guangxi, People's Republic of China

$\mathrm{Tel}+86 \quad 13978123845$

Fax +860771535003 I

Email jqzhang2002@I26.com
Purpose: Talaromyces marneffei (T.M) is an intracellular opportunistic fungus that causes invasive mycosis in patients with or without human immunodeficiency virus (HIV) infection. Hemophagocytic lymphohistiocytosis (HLH) caused by T.M infection is extremely rare. Here, we analyzed the clinical features, immune mechanisms, treatment, and prognosis related to this comorbidity.

Patients and Methods: This retrospective study was conducted between August 2012 and February 2019 at multiple research centers. Patients who presented with culture and/or histopathological proof of talaromycosis-associated HLH were included.

Results: HIV-negative patients $(\mathrm{n}=126)$ were enrolled. Of nine patients with T.M infection combined with secondary HLH, six were preschool children (five boys and one girl), and three were adults (two men and one woman). Seven of these nine had underlying diseases or recurrent infections. The most common symptoms were fever, anemia, hypoproteinemia, cough, weight loss, oral thrush, lymphadenopathy, hepatomegaly, splenomegaly, digestive symptoms, joint pain, and dyspnea. All patients showed reduced hemoglobin concentrations and platelet numbers. Liver dysfunction, hyperferritinemia, elevated lactate dehydrogenase, and low natural killer cell numbers were observed. Eight of nine patients received antifungal therapy, one patient did not receive therapy, and two of nine patients received anti-HLH therapy. Four died during treatment.

Conclusion: T.M fungemia associated with HLH was related to high mortality. Once diagnosed, timely and effective antifungal treatments and supportive care are essential.

Keywords: Talaromyces marneffei, hemophagocytic lymphohistiocytosis, human immunodeficiency virus, prognosis

\section{Introduction}

Talaromyces marneffei (T.M), formerly known as Penicillium marneffei, can cause talaromycosis marneffei (TSM), a systemic deep fungal disease. T.M can cause human infections in immunocompromised and healthy hosts. Recently, more cases of T.M infection have been described in human immunodeficiency virus (HIV)-negative individuals. Common clinical features of T.M infection among these non-HIV-infected patients include fever, malaise, weight loss, skin and soft tissue lesions, hepatosplenomegaly, lymphadenopathy, cough, and dyspnea. ${ }^{1}$ T.M infection is associated with high mortality because it is commonly 
misdiagnosed during the early stages of the disease. ${ }^{2}$ Moreover, secondary hemophagocytic lymphohistiocytosis $(\mathrm{SHLH}),{ }^{3}$ a rare clinical syndrome characterized by hyperinflammatory responses, high morbidity, acute onset, rapid progression, and high mortality, occurs as a comorbidity with other infections, particularly EpsteinBarr virus (EBV) and bacterial infections. ${ }^{4}$ However, SHLH has rarely been shown to be associated with TSM. Indeed, only three cases have been reported in the literature $;^{5-7}$ one case was in an HIV-negative patient, whereas the other two were HIV-positive cases. HLH is characterized by fever, cytopenia, splenomegaly, abnormal liver function, and hemophagocytosis (phagocytosis of erythrocytes, leukocytes, platelets, and their precursors by macrophages) in bone marrow. ${ }^{7}$ The clinical features of TSM are similar to those of HLH, clinicians are easy overlook, therefore, SHLH may be misdiagnosed as T.M infection deterioration, thereby affecting clinical outcomes. Accordingly, it is important to promote awareness of this comorbidity among clinicians.

In this study, we describe TSM associated with SHLH in nine patients without HIV. Our findings are expected to improve the rate of early recognition and facilitate the initiation of treatment.

\section{Materials and Methods}

\section{Study Population}

The medical records of 126 HIV-negative patients who were diagnosed with T.M infections between August 2012 and February 2019 at The First Affiliated Hospital of Guangxi Medical University, The Second Affiliated Hospital of Guangxi Medical University, and The Fourth People's Hospital were reviewed. Nine HIVnegative patients infected with T.M leading to SHLH were retrospectively evaluated. Data extracted from the medical records included demographic information (sex and age), clinical characteristics, laboratory findings, and clinical outcomes.

This study was approved by the Faculty of Medicine at The First Affiliated Hospital of Guangxi Medical University [2019(KY-E-061)]. All patients or patient's parents provided written informed consent. The study was carried out in accordance with the principles of the Declaration of Helsinki. The first author vouches for the completeness and accuracy of the data and for the fidelity of the study to the protocol.

\section{Inclusion and Exclusion Criteria}

Inclusion criteria were as follows: (i) HIV-negative; and (ii) a clear diagnosis of T.M infection with hemophagocytic lymphohistiocytosis. Patients diagnosed with T.M infection but who were HIV-positive were excluded.

\section{Determination of HIV Status}

Two enzyme-linked immunosorbent assays (EnzymunTest, Anti-HIV1+2; Boehringer Mannheim $\mathrm{GmbH}$ Diagnostica, Mannheim, Germany) were used to test the sera for the presence of anti-HIV antibodies. Any sera that were negative for anti-HIV antibodies underwent repeat testing at our hospital and at the Guangxi Centers for Disease Control and Prevention.

\section{Methods Used to Diagnose T.M Infection}

There were three methods used for pathological and pathogen examination. First, direct examination was performed. In this approach, direct smears of peripheral blood, bone marrow, skin lesions, and puncture fluid from marrow were all stained with Wright stain. Microscopic examination showed yeast-like cells with irregular sizes and shapes and clear septa. ${ }^{8}$ Second, cultures of clinical specimens (blood, bone marrow, sputum, and skin) were performed on Sabouraud's dextrose agar (SDA) at $25^{\circ} \mathrm{C}$ and $37^{\circ} \mathrm{C}$. Positive cultures for T.M were characterized as dimorphic fungi that grew as a mold at $25^{\circ} \mathrm{C}$ and as a yeast at $37^{\circ} \mathrm{C}$. A unique characteristic of the T.M mold is the presence of a soluble red pigment that diffuses into the agar, making the reverse side appear either pink or red at $25^{\circ} \mathrm{C}$. ${ }^{9}$ Finally, the yeast form of T.M was confirmed by cytological and histopathological assessments of tissue and secretion samples using Periodic Acid-Schiff or Wright staining, which revealed the characteristic morphology of intracellular yeast-like or sausage-like cells measuring 2-3 $\mathrm{mm}$ in diameter with transverse septa. ${ }^{10}$

\section{Diagnostic Criteria for HLH}

Patients were eligible if they met the 2004 Histiocyte Society Criteria for HLH diagnosis (HLH-2004). ${ }^{11}$ These criteria included a molecular diagnosis consistent with HLH and five of the following eight findings: fever $\left(\geq 38.5^{\circ} \mathrm{C}\right.$ for more than 7 days); splenomegaly; cytopenia affecting at least two of three lineages (i.e., hemoglobin $<90 \mathrm{~g} / \mathrm{L}$, platelet count $<100 \times 10^{9} / \mathrm{L}$, neutrophil count $<1.0 \times 10^{9} / \mathrm{L}$ ) ; hypertriglyceridemia $(\geq 3 \mathrm{mmol} / \mathrm{L}$ ) and/or hypofibrinogenemia 
$(\leq 1.5 \mathrm{~g} / \mathrm{L})$; hemophagocytosis in bone marrow, spleen, liver, or lymph nodes; hyperferritinemia $(\geq 500 \mu \mathrm{g} / \mathrm{L}$ ); low or absent natural killer (NK) cell cytotoxicity; and elevated level of serum-soluble CD25 (i.e., soluble interleukin-2 receptor $\geq 2400 \mathrm{IU} / \mathrm{mL}$ ).

\section{Results}

\section{Demographic Data and Clinical Characteristics}

During the 6-year study period, nine HIV-negative cases of T.M infection were evaluated at the hospital. All nine cases were born in Guangxi Province and had stayed in areas endemic for T.M in southern China. Patients who were diagnosed with TSM-associated SHLH satisfied the diagnostic criteria of HLH-2004. Six of nine cases were in preschool children (five boys and one girl; median age: 20 months [range: 17.3-33.5 months]). The median time from the onset of symptoms to diagnosis was 1.4 months (range: 0.9-2.5 months). The remaining three patients were adults (two men and one woman; median age: 42 years [range
40-49 years]). The median time from the onset of symptoms to diagnosis was 6.6 months (range: 6.4-9.1 months). Among the nine patients, six had underlying diseases or recurrent infection. The most common symptoms were fever and anemia. Seven patients had cough, six had weight loss, and two each had oral thrush, lymphadenopathy, hepatomegaly, and splenomegaly. One patient had digestive symptoms and joint pain, and dyspnea was also present (Table 1).

\section{Laboratory Examination}

All patients showed reduced hemoglobin concentrations and platelet counts. Complete blood count examinations revealed decreased white blood cell levels in seven patients $(77.8 \%)$ and decreased hemoglobin concentrations, platelet counts, and neutrophil counts in five patients. The median albumin level was $22.0 \mathrm{~g} / \mathrm{L}$, which was below the normal range for all patients (range: 20.6-23.3 g/L). Six patients (66.7\%) showed liver dysfunction. The median ferritin, triglycerides, and fibrinogen levels on presentation were $4451.9 \mathrm{ng} / \mathrm{mL}$ (range:

Table I Clinical Features of TSM-Associated SHLH

\begin{tabular}{|c|c|c|c|c|c|}
\hline No & $\begin{array}{l}\text { Age (Years) } / \\
\text { Sex }\end{array}$ & Occupation & Medical History & $\begin{array}{l}\text { Concurrent } \\
\text { Infection }\end{array}$ & Clinical Manifestations \\
\hline $\mathrm{PI}$ & $\mathrm{I} / \mathrm{M}$ & None & $\begin{array}{l}\text { Congenital megacolon, } \\
\text { oral thrush }\end{array}$ & No & Fever, cough, anemia, hepatosplenomegaly \\
\hline P2 & $2 / \mathrm{F}$ & None & Previously healthy & CMV, EBV & $\begin{array}{l}\text { Fever, cough, anemia, weight loss, edema of lower } \\
\text { extremity, hepatosplenomegaly }\end{array}$ \\
\hline P3 & $\mathrm{I} / \mathrm{M}$ & None & $\begin{array}{l}\text { Premature, growth } \\
\text { retardation }\end{array}$ & Thrush & $\begin{array}{l}\text { Fever, cough, anemia, weight loss, } \\
\text { hepatosplenomegaly, lymphadenopathy }\end{array}$ \\
\hline P4 & $\mathrm{I} / \mathrm{M}$ & None & Previously healthy & No & Fever, anemia, lymphadenopathy, hepatomegaly \\
\hline P5 & $4 / M$ & None & Frequent oral thrush & $\begin{array}{l}\text { Thrush, nail fungal } \\
\text { infection, EBV, } \\
\text { candidemia }\end{array}$ & $\begin{array}{l}\text { Fever, anemia, oral thrush, edema of lower extremity, } \\
\text { lymphadenopathy, hepatomegaly }\end{array}$ \\
\hline P6 & $\mathrm{I} / \mathrm{M}$ & None & $\begin{array}{l}\text { Premature low birth } \\
\text { weight, frequent } \\
\text { pneumonia }\end{array}$ & HBV & $\begin{array}{l}\text { Fever, cough, anemia, gastrointestinal symptom, } \\
\text { lymphadenopathy, hepatomegaly }\end{array}$ \\
\hline P7 & $42 / F$ & Farmer & Hyperthyroidism & Hepatitis B carrier & $\begin{array}{l}\text { Cough and sputum production, dyspnea, joint pain, } \\
\text { malaise, weight loss, skin lesion, lymphadenopathy }\end{array}$ \\
\hline P8 & $40 / M$ & Farmer & Previously healthy & No & $\begin{array}{l}\text { Fever, cough and sputum production, malaise, weight } \\
\text { loss, skin lesion, lymphadenopathy, } \\
\text { hepatosplenomegaly }\end{array}$ \\
\hline P9 & $49 / M$ & Farmer & SLE, lupus nephritis & No & $\begin{array}{l}\text { Fever, cough and sputum production, malaise, weight } \\
\text { loss, dental ulcer }\end{array}$ \\
\hline
\end{tabular}

Abbreviations: CMV, cytomegalovirus; EBV, Epstein-Barr virus; HBV, hepatitis B virus; SLE, systemic lupus erythematosus. 
Table 2 Laboratory Work-Up Results for TSM-Associated SHLH

\begin{tabular}{|c|c|c|c|c|c|c|c|c|c|c|}
\hline Variables & PI & $\mathbf{P 2}$ & $\mathbf{P 3}$ & P4 & P5 & P6 & P7 & P8 & P9 & Median (IQR) \\
\hline WBC $\left(\times 10^{9} / \mathrm{L}\right)$ & 6.7 & 6.6 & 3.5 & 1.7 & 4.8 & 4.4 & 1.9 & 2.2 & 3.9 & $3.7(2.1-5.7)$ \\
\hline ANC $\left(\times 10^{9} / \mathrm{L}\right)$ & 1.60 & 3.92 & 1.62 & 0.06 & 2.54 & 1.44 & 1.00 & 1.82 & 1.86 & $1.6(1.2-2.2)$ \\
\hline $\operatorname{ALC}\left(\times 10^{9} / \mathrm{L}\right)$ & 3.5 & 2.5 & 1.4 & 1.6 & 2.0 & 1.8 & 0.3 & 0.3 & 1.9 & $1.8(0.9-2.3)$ \\
\hline HGB $(g / L)$ & 77.0 & 62.3 & 82.6 & 84.0 & 79.1 & 81.0 & 72.0 & 67.8 & 64.3 & $77.0(66.1-81.8)$ \\
\hline PLT $\left(\times 10^{9} / L\right)$ & 15.0 & 18.2 & 87.5 & 25.4 & 22.3 & 66.0 & 93.0 & 4.6 & 78.0 & $25.4(16.6-82.8)$ \\
\hline TBiL (mmoL/L) & 9.4 & 11.2 & 47.1 & 14.6 & 18.9 & 8.5 & 19.6 & 10.7 & 37.9 & $14.6(10.1-28.8)$ \\
\hline DBiL (mmoL/L) & 4.1 & 4.9 & 38.8 & 6.2 & 16.4 & 2.9 & 12.0 & 7.5 & 25.3 & $7.5(4.5-20.9)$ \\
\hline Albumin (g/L) & 24.1 & 20.4 & 19.1 & 22.0 & 22.2 & 26.5 & 20.7 & 21.9 & 22.5 & $22.0(20.6-23.3)$ \\
\hline ALT (U/L) & 22 & 193 & 362 & 449 & 113 & 68 & 26 & 9 & 144 & $40(16-111)$ \\
\hline AST (U/L) & 13 & 40 & 108 & 59 & 737 & 18 & 23 & 11 & 677 & $144(24-405)$ \\
\hline LDH (U/L) & 481 & 810 & 396 & 2894 & 2609 & 312 & 296 & 906 & 3056 & 810 (354-2752) \\
\hline FIB $(g / L)$ & 2.44 & 0.41 & 0.26 & ND & 0.79 & 0.94 & 1.79 & 6.27 & 0.69 & $0.87(0.48-2.28)$ \\
\hline PT $(s)$ & 28.7 & 24.9 & 35.0 & 14.0 & $4 \mathrm{I} .4$ & 12.2 & 23.9 & 21 & 13.4 & $23.9(|3.7-3| .9)$ \\
\hline TG (mmol/L) & 1.13 & 3.31 & 2.15 & ND & 4.98 & 4.95 & ND & ND & 10.44 & $4.1(1.9-6.3)$ \\
\hline Ferritin $(\mathrm{ng} / \mathrm{mL})$ & 5992.9 & 4451.9 & 1647.0 & 1312.7 & $18,327.9$ & 792.3 & 747.4 & $46,020.0$ & 8966.4 & $4451.9(1052.5-13,647.1)$ \\
\hline $\mathrm{DD}(\mathrm{ng} / \mathrm{mL})$ & 1763 & 5851 & ND & ND & 9842 & 4184 & 637 & 4075 & 18,473 & $9842(4075-14,004)$ \\
\hline
\end{tabular}

Notes: Normal ranges: absolute neutrophil count: I.80-6.30 × 10\%/L; absolute lymphocyte count: I.I-3.2 × $10^{9} / \mathrm{L}$; glycerin trilaurate: $0.5-1.70$ mmoL/L; fibrinogen: $2-5$ g/L; ferritin: 21.8-274.66 ng/mL; D-dimer: 0-450 ng/mL.

Abbreviations: WBC, white cell count; Neu, neutrophil; ALC, absolute lymphocyte count; HGB, hemoglobin; PLT, platelet; FIB, fibrinogen; LDH, lactate dehydrogenase; PT, prothrombin time; TG, triglycerides; DD, D-dimer; ND, not done.

1052.5-13,647.1 $\mathrm{ng} / \mathrm{mL}$ ), $4.1 \mathrm{mmoL} / \mathrm{L}$ (range: 1.9-6.3 $\mathrm{mmol} / \mathrm{L}$ ), $0.87 \mathrm{~g} / \mathrm{L}$ (range: 0.48-2.28 g/L), respectively, and $66.7 \%$ of patients had increased prothrombin times (Table 2).

\section{Inflammation and Immunological Indexes}

C-reactive protein concentrations and erythrocyte sedimentation rates were increased in all patients. Four showed reduced globulin, and serum IgG levels were increased in three patients $(37.5 \%)$. Notably, patient 4 showed decreases in serum globulin, serum IgG, IgA, and IgM. Two patients $(50.0 \%)$ showed significant reductions in $\mathrm{CD}^{+} \mathrm{T}$-cell counts and $\mathrm{CD}^{+} \mathrm{T}$-cell counts. Six of seven patients $(85.7 \%)$ showed low NK cell counts (Table 3).

\section{Chest Radiography and Computed Tomography (CT)}

Chest CT indicated that all patients had different pulmonary lesions. Four (44.4\%) had diffused patchy density shadow. Seven (77.8\%) had pleural inflammatory reaction and/or pleural effusion, and six (66.7\%) had mediastinal and/or hilar lymphadenopathy. Two $(22.2 \%)$ had pericardial effusion, cavities, pulmonary consolidation, and osteolytic lesions in the ribs (Figure 1A and B).

\section{Fungal Culture and Histopathology}

Fluid was aspirated from the bone marrow, blood, pleural effusion, bronchoalveolar lavage fluid, and dermal secretions. Samples were then inoculated onto SDA and incubated at $37^{\circ} \mathrm{C}$ or $25^{\circ} \mathrm{C}$. Nine cases were confirmed to be positive for T.M culture. T.M was isolated from venous blood $(6 / 9,66.7 \%)$, bone marrow $(3 / 5,60 \%)$, sputum samples $(2 / 5,40 \%)$, and dermal lesion secretions $(2 / 2,100 \%)$. In addition, three cases were diagnosed with T.M infection by histopathology or cytology of specimens obtained from bone marrow $(2 / 7,28.6 \%)$ or lymph nodes $(1 / 1,100 \%)$. Bone marrow aspirate from three patients (3/7, 42.9\%) showed histiocytic hyperplasia and marked hemophagocytosis (Figure 2A and B), and all bone marrow analyses showed the absence of leukemia.

\section{Diagnosis of TSM and HLH}

Diagnosis of HLH requires fulfillment of the criteria described in the Methods. Patient 7 was progressing rapidly, and some examinations were not performed (Table 4).

\section{Treatments and Outcomes}

Antifungal therapy was administered to eight patients immediately after the diagnosis of TSM; however, one patient died of disseminated intravascular coagulopathy before the diagnosis of T.M infection, and antifungal therapy was not administered. Patients 4 and 7, who received 
Table 3 Inflammation and Immunological Indexes of TSM-Associated SHLH

\begin{tabular}{|c|c|c|c|c|c|c|c|c|c|}
\hline Variables & $\mathbf{P I}$ & $\mathbf{P 2}$ & P3 & P4 & P5 & P6 & P7 & P8 & P9 \\
\hline CRP (mg/L) & 40.5 & 78.28 & $>192$ & 131.5 & 98.57 & $>192$ & 101.05 & 94.63 & 38.99 \\
\hline $\mathrm{ESR}(\mathrm{mm} / \mathrm{h})$ & ND & ND & ND & ND & ND & 7 & 92 & 124 & $>140$ \\
\hline PCT (ng/L) & ND & 8.76 & ND & 30.27 & 36.35 & 3.04 & ND & 100 & ND \\
\hline G-test (pg/mL) & 15.16 & ND & ND & $5 I 5.9 \uparrow$ & ND & 65.7 & ND & $<10$ & ND \\
\hline GM-test & ND & ND & ND & ND & ND & $3.782 \uparrow$ & $0.645 \uparrow$ & 0.329 & ND \\
\hline Globulin (g/L) & 21.7 & $46.1 \uparrow$ & 32.0 & $9.5 \downarrow$ & 24.7 & 25.8 & $63.4 \uparrow$ & $55.0 \uparrow$ & $56.3 \uparrow$ \\
\hline $\lg G(g / L)$ & $|5.3|$ & $26.90 \uparrow$ & 16.80 & $0.19 \downarrow$ & 14.78 & 10.21 & $40.07 \uparrow$ & ND & $20.75 \uparrow$ \\
\hline $\operatorname{lgA}(g / L)$ & $\mathrm{I} .48$ & 1.33 & 1.39 & $0.03 \downarrow$ & 2.01 & $0.7 I \downarrow$ & 3.78 & ND & $5.15 \uparrow$ \\
\hline $\lg M(g / L)$ & 0.87 & 0.95 & $3.77 \uparrow$ & $0.48 \downarrow$ & $\mathrm{I} .33 \uparrow$ & $\mathrm{I} .4 \mathrm{I} \uparrow$ & $1.96 \uparrow$ & ND & $2.99 \uparrow$ \\
\hline C3 (g/L) & ND & $0.592 \downarrow$ & ND & ND & $0.073 \downarrow$ & $\mathrm{I} .357$ & ND & ND & ND \\
\hline C4 $(g / L)$ & ND & $0.136 \downarrow$ & ND & ND & $0.022 \downarrow$ & 0.286 & ND & ND & ND \\
\hline $\mathrm{CD}^{+} \mathrm{T}$ cell count (cells $\left./ \mu \mathrm{L}\right)$ & ND & ND & ND & 436 & ND & $4288 \uparrow$ & ND & $299 \downarrow$ & $95 \downarrow$ \\
\hline $\mathrm{CD}^{+} \mathrm{T}$ cell count (cells $\left./ \mu \mathrm{L}\right)$ & ND & ND & ND & 1044 & ND & $1219 \uparrow$ & ND & $|5| \downarrow$ & $133 \downarrow$ \\
\hline CD4\% & $22.2 \downarrow$ & ND & 39.1 & ND & $27.5 \downarrow$ & $60.2 \uparrow$ & ND & 34.2 & $0.7 I \downarrow$ \\
\hline CD8\% & 29.4 & ND & $16.9 \downarrow$ & ND & 27.9 & $8.5 \downarrow$ & ND & $36.8 \uparrow$ & ND \\
\hline $\mathrm{T}$ cell\% & $54.1 \downarrow$ & ND & $63.7 \downarrow$ & ND & $59.2 \downarrow$ & 70.0 & ND & 74.6 & $79.5 \uparrow$ \\
\hline NK\% & $2.1 \downarrow$ & $2.8 \downarrow$ & $\mathrm{I} .53 \downarrow$ & ND & $2.3 \downarrow$ & 9.5 & ND & $3.2 \downarrow$ & $4.5 \downarrow$ \\
\hline
\end{tabular}

Notes: Normal ranges: C-reactive protein: $<10 \mathrm{mg} / \mathrm{L}$; erythrocyte sedimentation rate: $\leq 15 \mathrm{~mm} / \mathrm{h}$; procalcitonin: $<0.05 \mathrm{ng} / \mathrm{L} ;$ elevated $(\mathrm{I} \rightarrow 3)-\beta-\mathrm{d}$-glucan: $<100 \mathrm{pg} / \mathrm{mL}$; Aspergillus galactomannan antigen: < 0.5; immunoglobulin (Ig) IgG: 8-18 g/L; IgA: 0.9-4 g/L; IgM: 0.84-I.32 g/L; CD4 + T cell count: 4I0-I590 cells/ $\mu \mathrm{L}$; CD8 ${ }^{+}$T cell count: 190-II40 cells/ $\mu$ L; T lymphocytes: 64.2-78.5\%; CD4\%: 30.I-40.4\%; CD8\%: 20.7-29.4\%; NK\%: 9-I5\%; C3: 0.79-I.52 g/L; C4: 0.16-0.38 g/L. G-test elevated (I-3)- $\beta-$ d-glucan.

Abbreviations: CRP, C-reactive protein; ESR, erythrocyte sedimentation rate; GM-test, Aspergillus galactomannan antigen; IgG:, serum immunoglobulin G; IgA, serum immunoglobulin A; IgM, serum immunoglobulin M; NK, natural killer cells.

intravenous fluconazole, died of heart failure or septic shock within 2 weeks. Patient 9 was treated with intravenous fluconazole showed no recurrence after 1 week. He then received voriconazole, and the clinical symptoms improved after 2 weeks. Five patients received voriconazole, one of whom died during treatment. The remaining four patients had their temperatures return to normal, with significant improvement of their clinical symptoms a median of 13 days (range: 4-19 days) after antifungal therapy. Patients were treated with oral voriconazole or itraconazole, and all four patients showed improvement 4-24 months later during follow-up. In addition, no relapse was observed during the 2-year period of antifungal treatment. However, patient 1 died of leukemia. Eight of nine patients received a specific treatment for HLH after diagnosis; treatment included corticosteroids (CS) and/or
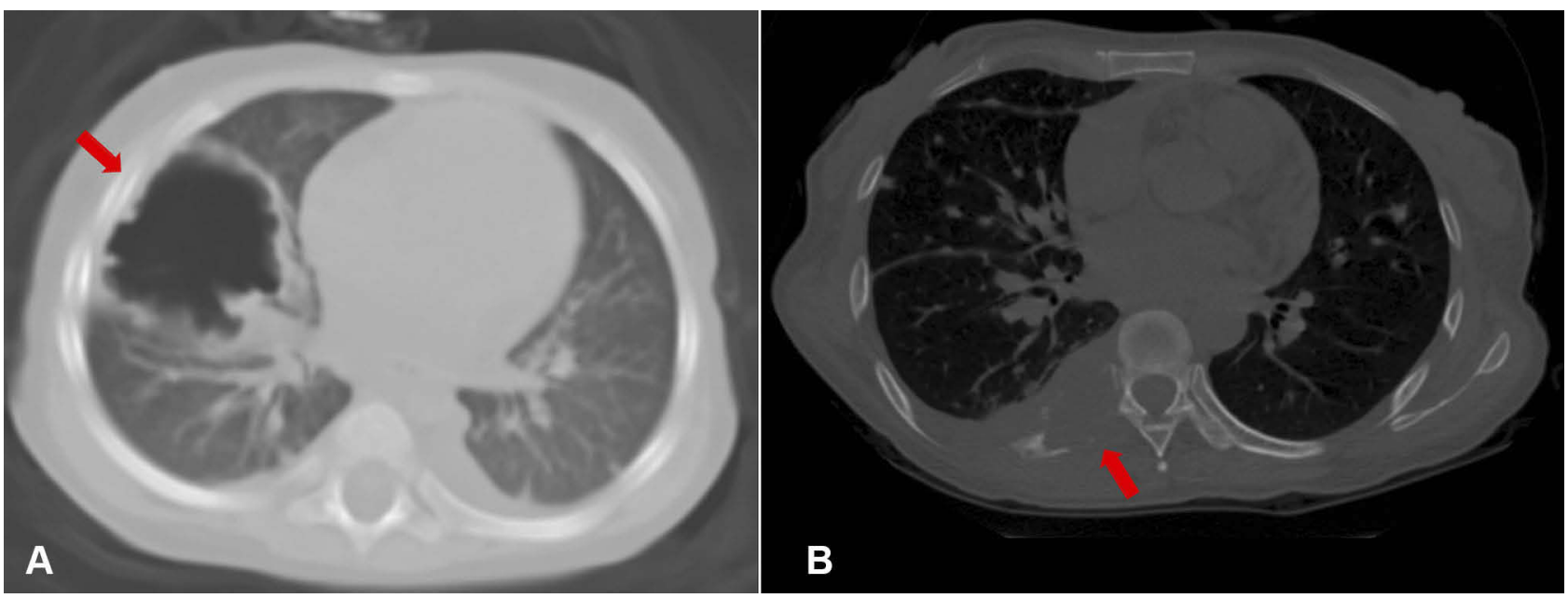

Figure I High-resolution computed tomography. High-resolution computed tomography showing a cavitary lesion (arrow) (A) and osteolytic lesions in the ribs accompanied by soft tissue swelling (B). 


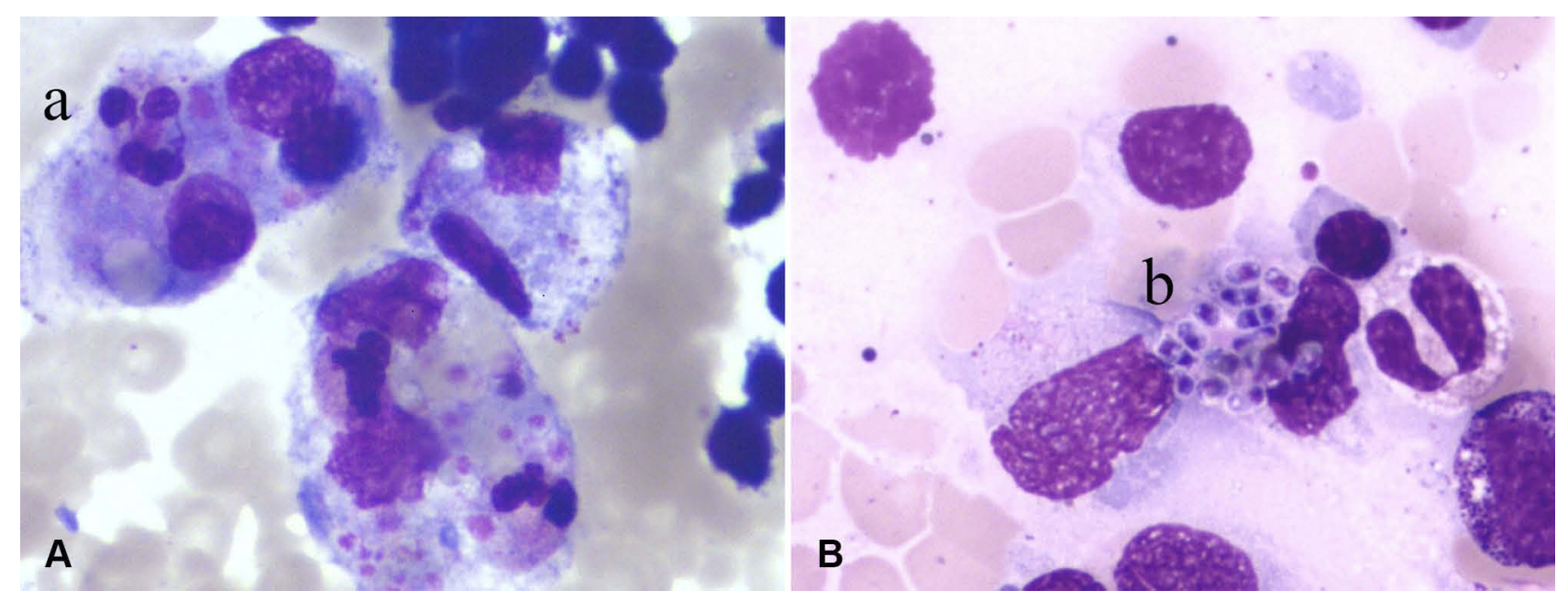

Figure 2 (A) Bone marrow aspirate with phagocytosed erythroid cells and a neutrophil-e granulocyte (a) (magnification: I,000x). (B) Periodic acid-Schiff staining of numerous intracellular and extracellular microorganisms with distinct central septa (b) (magnification: I,000x).

intravenous immunoglobulin (IVIG) in six patients, CS + etoposide + IVIG in two patients. One patient received supportive care without anti-HLH treatment (Table 5).

\section{Discussion}

T.M is an important pathogenic thermally dimorphic fungus that causes systemic mycosis in Southeast Asia countries and the southern part of China. ${ }^{12}$ T.M infection occurs not only in HIV-infected patients but also in both immunocompetent and immunocompromised patients without HIV, such as patients with hyperimmunoglobulin $\mathrm{M}$ syndrome, hyperimmunoglobulin E syndrome, ${ }^{13}$ hypogammaglobulinemia,${ }^{14}$ adult-onset immunodeficiency syndrome caused by anti-interferon (IFN)- $\gamma$ autoantibodies, ${ }^{15,16}$ solid organ transplantation and

Table 4 Diagnosis of TSM and HLH

\begin{tabular}{|c|c|c|}
\hline Patient & $\begin{array}{l}\text { Site(s) of Positive } \\
\text { Culture Histology }\end{array}$ & Diagnostic Criteria for HLH \\
\hline PI & Blood, BM smear & $\begin{array}{l}\text { (i) Tmax } 40.2^{\circ} \mathrm{C} \text {, (ii) splenomegaly, (iii) peripheral blood cytopenia for two lineages, (iv) } \\
\text { hemophagocytosis in bone marrow, (v) low NK cell activity }\end{array}$ \\
\hline P2 & Blood, BM & $\begin{array}{l}\text { (i) } \operatorname{Tmax} 39.8^{\circ} \mathrm{C} \text {, (ii) splenomegaly, (iii) peripheral blood cytopenia for two lineages, (iv) FIB }<1.5 \mathrm{~g} / \mathrm{L} \text {, (v) } \\
\text { low NK cell activity }\end{array}$ \\
\hline P3 & Blood & $\begin{array}{l}\text { (i) Tmax } 38.7^{\circ} \mathrm{C} \text {, (ii) splenomegaly, (iii) peripheral blood cytopenia for two lineages, (iv) low NK cell } \\
\text { activity, (v) ferritin }>500 \mathrm{ng} / \mathrm{mL}\end{array}$ \\
\hline P4 & Blood & $\begin{array}{l}\text { (i) Tmax } 39.0^{\circ} \mathrm{C} \text {, (ii) splenomegaly, (iii) peripheral blood cytopenia for three lineages, (iv) FIB }<1.5 \mathrm{~g} / \mathrm{L} \text {, } \\
\text { (v) ferritin }>500 \mathrm{ng} / \mathrm{mL}\end{array}$ \\
\hline P5 & Blood, sputum, BM smear & $\begin{array}{l}\text { (i) Tmax } 39.0^{\circ} \mathrm{C} \text {, (ii) splenomegaly, (iii) peripheral blood cytopenia for two lineages, (iv) FIB }<1.5 \mathrm{~g} / \mathrm{L} \text { and } \\
\mathrm{TG}>3 \mathrm{mmol} / \mathrm{L} \text {, (v) hemophagocytosis in bone marrow, (vi) low NK cell activity, (vii) ferritin }>500 \mathrm{ng} / \mathrm{mL}\end{array}$ \\
\hline P6 & Blood, BM & $\begin{array}{l}\text { (i) } \operatorname{Tmax} 39.5^{\circ} \mathrm{C} \text {, (ii) splenomegaly, (iii) peripheral blood cytopenia for two lineages, (iv) FIB < } 1.5 \mathrm{~g} / \mathrm{L} \text {, (v) } \\
\text { ferritin }>500 \mathrm{ng} / \mathrm{mL}\end{array}$ \\
\hline P7 & Skin & (i) $\operatorname{Tmax} 4 \mathrm{I}^{\circ} \mathrm{C}$, (ii) peripheral blood cytopenia for two lineages, (iii) $\mathrm{FIB}<1.5 \mathrm{~g} / \mathrm{L}$, (iv) ferritin $>500 \mathrm{ng} / \mathrm{mL}$ \\
\hline P8 & Skin, LN & $\begin{array}{l}\text { (i) Tmax } 39.0^{\circ} \mathrm{C} \text {, (ii) splenomegaly, (iii) peripheral blood cytopenia for two lineages, (iv) low NK cell } \\
\text { activity, (v) ferritin }>500 \mathrm{ng} / \mathrm{mL}\end{array}$ \\
\hline P9 & BM, sputum & $\begin{array}{l}\text { (i) Tmax } 39.1^{\circ} \mathrm{C} \text {, (ii) peripheral blood cytopenia for two lineages, (iii) TG }>3 \mathrm{mmol} / \mathrm{L} \text {, (iv) low NK-cell } \\
\text { activity, (v) ferritin }>500 \mathrm{ng} / \mathrm{mL}\end{array}$ \\
\hline
\end{tabular}

Abbreviations: BM, bone marrow; LN, lymph node; FIB, fibrinogen; Tmax, Maximum temperature. 
Table 5 Treatment of TSM-Associated SHLH

\begin{tabular}{|c|c|c|c|c|}
\hline Patient & TSM Therapy & HLH Therapy & TSM Period of Treatment & Outcome \\
\hline $\mathrm{PI}$ & VCZ $7 \mathrm{mg} / \mathrm{kg}$ every $12 \mathrm{~h}$ & $\begin{array}{l}\text { Dexamethasone } 10 \mathrm{mg} / \mathrm{m}^{2} \text { for } 5 \text { days + IVIG } \\
2.5 \mathrm{~g} \text { for } 2 \text { days }\end{array}$ & $\begin{array}{l}\text { VCZ } 20 \text { weeks, recurrence after } 20 \text { days } \\
\text { of withdrawal, VCZ } 2 \text { years, diagnosis } \\
\text { leukemia died after } 3 \text { months }\end{array}$ & Death \\
\hline P2 & VCZ $6.5 \mathrm{mg} / \mathrm{kg}$ every $12 \mathrm{~h}$ & $\begin{array}{l}\text { Standard treatment protocol for HLH } \\
\text { consisting of dexamethasone and etoposide } \\
+ \text { IVIG } 2.5 \mathrm{~g} \text { for } 5 \text { days }\end{array}$ & VCZ for 2 years & Improve \\
\hline P3 & None & $\begin{array}{l}\text { Dexamethasone } 10 \mathrm{mg} / \mathrm{m}^{2} \text { for } 3 \text { days + IVIG } \\
2.5 \mathrm{~g} \text { for } 2 \text { days }\end{array}$ & None & Death \\
\hline P4 & $\mathrm{FLC} 3 \mathrm{mg} / \mathrm{kg} /$ day & IVIG $2.5 \mathrm{~g}$ for 2 days & None & Death \\
\hline P5 & VCZ $7 \mathrm{mg} / \mathrm{kg}$ every $12 \mathrm{~h}$ & $\begin{array}{l}\text { Etoposide once }+ \text { dexamethasone } 10 \mathrm{mg} / \mathrm{m}^{2} \\
\text { for } 4 \text { days }+ \text { IVIG } 2.5 \mathrm{~g} \text { for } 2 \text { days }\end{array}$ & None & Death \\
\hline P6 & VCZ $4.5 \mathrm{mg} / \mathrm{kg}$ every $12 \mathrm{~h}$ & $\begin{array}{l}\text { MTH I5 mg for } 4 \text { days + IVIG } 2.5 \mathrm{~g} \text { for } 3 \\
\text { days }\end{array}$ & VCZ for 5 months & Improve \\
\hline P7 & FLC 400 mg/day & None & None & Death \\
\hline P8 & VCZ $200 \mathrm{mg}$ every $12 \mathrm{~h}$ & Dexamethasone $15 \mathrm{mg} /$ days for 2 weeks & Lost to follow-up & Unknown \\
\hline P9 & $\begin{array}{l}\text { FLC } 200 \mathrm{mg} / \text { day for I week } \\
\text { then change to VCZ } \\
\text { improved after } 2 \text { weeks }\end{array}$ & $\begin{array}{l}\text { MTH } 40 \mathrm{mg} \text { for } 2 \text { days, } 80 \mathrm{mg} \text { for } 7 \text { days, } \\
60 \mathrm{mg} \text { for } 7 \text { days, IVIG I5 mg for } 4 \text { days, I } \\
\text { week after IVIG I } 5 \mathrm{mg} / 2 \text { days }\end{array}$ & ITC for 4 months & Improve \\
\hline
\end{tabular}

Abbreviations: IV, intravenous; VCZ, voriconazole; FLC, fluconazole; ITC, itraconazole; IVIG, intravenous immunoglobulins; DIC, disseminated intravascular coagulopathy; MTH, methylprednisolone.

hematopoietic stem cell transplantation recipients, and hematology patients who have been treated with novel targeted therapies, including anti-CD20 monoclonal antibodies and kinase inhibitors. ${ }^{17}$ However, T.M infection associated with HLH is extraordinarily rare, and only three prior cases have been reported; one case occurred in an HIV-negative patient with Sjogren's syndrome treated with prednisolone and cyclophosphamide, ${ }^{5}$ whereas the other two (one a child ${ }^{6}$ and one an adult ${ }^{7}$ ) were HIV-positive. To the best of our knowledge, this is the first study reporting the clinical features and progress of TSM-associated SHLH.

The nine patients described in this study were all HIV-negative; six of these nine were preschool children, who showed common growth dysplasia and recurrent bacterial or viral infections. Three were adults, two of whom had underlying diseases, such as hyperthyroidism or systemic lupus erythematosus. These findings suggested that patients with severe immunodeficiencies or immune abnormalities were at higher risk of T.M infection. T.M infection in HIV-negative children may be related to immunodeficiencies, such as Th17 deficiency, autosomaldominant gain-of-phosphorylation signal transducer and activator of transcription (STAT) 1 mutations, and heterozygous missense mutations in exon 12 of the STAT3 gene. Analysis of STAT1 and STAT3 mutant genes showed that Th1 and Th17 immune responses play important roles in host infection with T.M. ${ }^{13,16}$ In this study, patient 5 had a medical history of frequent oral thrush, and patient 4 died of heart failure, severe multiple organ failure, hypogammaglobulinemia, and agranulocytosis. However, in this patient, there was no conclusive diagnosis of immunodeficiency, suggesting that the patients may have undefined severe cellular immune dysfunction. In adults, even HIV-negative patients with TSM, without any underlying diseases, are likely to develop a new type of adult immunodeficiency syndrome owing to the presence of anti-IFN- $\gamma$ autoantibodies. This can be the cause of cell-mediated immunity defects in HIV-negative adults. The pathogenesis of T.M infection associated with SHLH may involve severe inflammatory response syndrome caused by congenital or postinfection immune deficiency or by severe infection. The imbalance of immunomodulation, accumulation of immunocompetent cells, and production of inflammatory cytokines are key factors in the pathogenesis of HLH. ${ }^{17}$ Animal models have been shown to play key roles in IFN- $\gamma$ production by $\mathrm{CD}^{+} \mathrm{T}$ lymphocytes in the pathophysiology of 
HLH. ${ }^{18} \mathrm{CD}^{+} \mathrm{T}$ lymphocytes mediate immunodeficiency to play key roles in HIV-positive patients. ${ }^{19}$ Importantly, in our study, $\mathrm{CD}^{+} \mathrm{T}$ cells, $\mathrm{CD} 8^{+} \mathrm{T}$ cells, and immunoglobulin were also abnormal but gradually became normal as the disease status improved, suggesting that T.M infection could lead to immune dysfunction. Prior literature and our study showed that HLH in HIV-negative children patients with T.M infection was more common than that in HIVpositive patients. However, the mechanisms and diagnosis of adult-onset HLH are poorly understood, and further studies are needed to improve our understanding of these topics.

It is difficult to differentiate between T.M fungemia and HLH owing to their similar manifestations. According to the literature, when TSM occurs in HIV-negative hosts, white blood cells are increased, reaching up to $57.48 \times 10^{9} / \mathrm{L}^{20}$ Therefore, when TSM is diagnosed, white blood cell levels are typically low. Alternatively, when patients exhibit the rapid onset of pancytopenia, relevant examinations should be performed in time to identify possible SHLH. The diagnostic criterion of HLH includes neutrophils of less than $1 \times 10^{9} / \mathrm{L}$; if neutrophils are reduced to granulocyte deficiency, the infection is often severe, and the prognosis is poor, as was observed for patient 4 . In addition, the platelet count of the peripheral blood is also an index that needs to evaluate at an early stage. When patients with TSM exhibit acute platelet decline, clinicians should consider excluding SHLH. Increases in serum ferritin, lactate dehydrogenase, and liver enzymes are also useful indexes for the diagnosis of HLH. ${ }^{21}$ The presence of nucleated red blood cells in peripheral blood on bone marrow smears usually indicates bone marrow infiltration in the absence of serious hemolysis, hemorrhage, or sepsis. ${ }^{22}$ Hemophagocytic cells can be found by multisite bone marrow puncture when patients with TSM exhibit whole blood cell reduction, elevated ferritin levels, and hypertriglyceridemia. Even if no hemophagocytic cells were found in bone marrow, HLH could be diagnosed. All of our patients satisfied the diagnostic criteria for HLH-2004, although not all of their bone marrow smears contained hemophagocytes. Compared with adults, the incidence of hepatosplenomegaly in children is higher. ${ }^{10}$ When T.M infection leads to SHLH, splenomegaly may be caused by reactive proliferation in the spleen as well as T.M invasion of the spleen. These findings may be explored further to identify the etiology of T.M.
Our patients had the highest culture-positive rates in blood, indicating that the infection could become severe and spread hematogenously to other organs, potentially leading to severe inflammatory reactions. Invasion of monocyte-macrophage systems, particularly the blood, bone marrow, liver, spleen, lung, lymph nodes, and skin, by infection can cause multiple organ failure, abnormal proliferation of histiocytes, and even myelosuppression. Combined with gradual decreases in platelets and hemoglobin, similar to symptoms observed in HLH, patients may eventually die of disseminated intravascular coagulation (DIC).

Talaromycosis leading to SHLH, particularly in patients with underlying diseases, runs a rapid progressive course with high mortality rates, and early application of effective antifungals is an important measure for improving prognosis. In the nine patients in our study with TSM-associated SHLH, five died (mortality rate of 55.6\%) during treatment; the causes of death were leukemia, heart failure, sepsis, DIC, and septic shock. The findings from previous studies have shown that amphotericin $\mathrm{B}$, itraconazole, and fluconazole are useful antifungal options for T.M infection. ${ }^{23}$ In our study, eight cases were treated with antifungal therapy, among which two were initially treated with fluconazole; these patients died of heart failure or septic shock within 2 weeks. The initial use of fluconazole in patient 9 was ineffective for 1 week, and the patient then received voriconazole, resulting in improvement of condition after 2 weeks of replacement. Fluconazole, although effective in the initial treatment, can easily lead to resistance and should not be the first choice. ${ }^{24}$ Because of the low nephrotoxicity, high sensitivity, and high efficacy of voriconazole, intravenous voriconazole could be recommended as the initial antifungal agent for infants. Five cases were treated with intravenous voriconazole. In addition, the clinical symptoms were improved, and the final oral administration of voriconazole or itraconazole was completed after 4-24 months, resulting in clinical cure. In patients with defective immune systems, compared with itraconazole, amphotericin B treatment shows faster clinical efficacy, enhanced fungal clearance, and significantly lower recurrence rates. ${ }^{25}$ However, regardless of what type of initial treatment is used, in the absence of clinical effects within 7 days, antifungal therapy should be replaced, and amphotericin B can be considered if necessary. We have reported an HIV-negative patient who relapsed after 4 years; therefore, for immunodeficiencies, longer-term secondary prophylaxis and follow-up may be necessary, the withdrawal 
of drugs should be based on the complete disappearance of clinical symptoms and signs, normal imaging examination, and, more importantly, the return of immune function to normal. Infection-associated HLH can result in poor outcomes. Predictors of a poor prognosis include age older than 30 years, the presence of disseminated intravascular coagulation, anemia with accompanying thrombocytopenia, and elevated ferritin level. ${ }^{22}$ Indeed, patient 5 died of serum ferritin levels greater than $10,000 \mathrm{ng} / \mathrm{mL}$ and DIC.

There is still no unified standard regarding whether patients with TSM-associated SHLH should receive standardized chemotherapy. The treatment duration is typically 8 weeks in patients with HLH who do not have an identifiable genetic defect. ${ }^{22}$ In this study, treatments were CS and/ or IVIG in six patients and CS + etoposide + IVIG in two patients. One patient received supportive care without antiHLH treatment and finally died. In a review of three cases of TSM-associated SHLH, only IVIG and CS therapy were given to patients with HLH, and after a full course of antifungal therapy, the condition was improved. ${ }^{5-7}$ Three patients in this group were given active antifungals, CS, and IVIG after diagnosis. Only one case was cured clinically using a combination of an antifungal combined with etoposide. After infection with T.M, the immune function of the body is suppressed, and chemotherapeutic drugs may lead to further aggravation of bone marrow or immunosuppression, which is disadvantageous for controlling the infection and could aggravate the risk of spread of the infection. Importantly, small amounts of short-term hormones are beneficial for controlling inflammatory responses caused by T.M infection. If effective antifungals are given, but bone marrow suppression, peripheral blood leukocytes, platelets, anemia, and other blood cell counts do not improve significantly, it may be effective to attempt the HLH-2004 chemotherapy regimen. HLH is a serious complication of T. $M$ infection, and antifungal therapy is an important approach for controlling the disease. Thus, application of antifungal therapy should be an acceptable course of treatment; however, in HIV-negative hosts, TSM may recur because of the complexity of the immune deficiency mechanism, and a longer treatment duration may be needed. In areas endemic for T.M infection, when patients are diagnosed with HLH, after excluding viral infection and other types of infections, infection caused by T.M must be considered. Moreover, the etiological basis of infection should be analyzed, particularly in children. Once diagnosed, clinicians should take time to identify the underlying mechanisms of immune deficiency.
One limitation of this study is that we did not perform a complete immunological study to identify all possible immunological abnormalities in these patients because this was a retrospective study. More studies are needed to address key questions regarding the use of prospective surveillance and optimal treatment strategies.

\section{Conclusions}

T.M infection leading to HLH is extremely rare and associated with a high mortality rate. However, it is difficult to differentiate between T.M fungemia and HLH. Thus, when patients are diagnosed with HLH, after excluding viral infections and other types of infections, infection caused by T.M must be considered. Once diagnosed, clinicians should carefully consider the mechanisms of immune deficiency, and timely, effective antifungal treatments and supportive care are essential.

\section{Ethical Approval and Informed Consent}

This study was approved by the ethics committee associated with the Faculty of Medicine at The First Affiliated Hospital of Guangxi Medical University [2019(KYE-061)]. Written informed consent was obtained from the patients for publication of this article and any accompanying images. Copies of the written consents are available for review.

\section{Author Contributions}

M. Pan and Y. Qiu designed the study and analyzed data. M. Pan wrote a draft of the manuscript. W. Zeng, S. Tang, and X. Feng contributed to data collection. J. Zhang was responsible for critical revision of the manuscript. X. Wei, J. Deng, and Z. He helped perform the analysis and was involved in critical discussions. All authors contributed to data analysis, drafting or revising the article, gave final approval of the version to be published, and agree to be accountable for all aspects of the work.

\section{Funding}

This work was supported by the National Natural Science Foundation of China (grant nos. 81460009 and 81760010).

\section{Disclosure}

The authors declare no conflicts of interest associated with this paper. 


\section{References}

1. Chan JF, Lau SK, Yuen KY, Woo PC. Talaromyces (Penicillium) marneffei infection in non HIV-infected patients. Emerg Microbes Infections. 2016;5(3):e19. doi:10.1038/emi.2016.18

2. Qiu Y, Zhang JQ, Pan ML, et al. Determinants of prognosis in Talaromyces marneffei infections with respiratory system lesions. Chin Med J. 2019;132:16. doi:10.1097/CM9.0000000000000345

3. Jan-Inge H, Annacarin SH, Maurizio A, et al. Treatment of hemophagocytic lymphohistiocytosis with HLH-94 immunochemotherapy and bone marrow transplantation. An Pediatr (Barc). 2002;100 (7):2367. doi:10.1182/blood-2002-01-0172

4. George MR. Hemophagocytic lymphohistiocytosis: review of etiologies and management. J Blood Med. 2014;5:69-86. doi:10.2147/JBM

5. Chim CS, Fong CY, Ma SK, Wong SS. Reactive hemophagocytic syndrome associated with penicillium marneffei infection. Am J Med. 1998;104(2):196. doi:10.1016/S0002-9343(97)00253-2

6. Chokephaibulkit K, Veerakul G, Vanprapar N, Chaiprasert A. Penicilliosis-associated hemophagocytic syndrome in a human immunodeficiency virus-infected child: the first case report in children. J Med Assoc Thai. 2001;84(3):426-429.

7. Pei S, Lee CJ. Hemophagocytic syndrome in a patient with acquired immunodeficiency syndrome and acute disseminated penicilliosis. Am J Trop Med Hyg. 2008;78(1):11-13. doi:10.4269/ajtmh.2008. 78.11

8. Xi L, Lu C, Zhou X, Wang L, Xie SJM. Fifteen cases of penicilliosis in Guangdong, China.Mycopathologia.2004;158(2):151-155. doi:10.1023/ B:MYCO.0000041842.90633.86

9. Sirisanthana T, Supparatpinyo K. Epidemiology and management of penicilliosis in human immunodeficiency virus-infected patients. Int $J$ Infect Dis. 1998;3(1):48-53. doi:10.1016/S1201-9712(98)90 095-9

10. Zeng W, Qiu Y, Lu D, Zhang J. A retrospective analysis of 7 human immunodeficiency virus-negative infants infected by Penicillium marneffei. Medicine (Baltimore). 2015;94(34):e1439. doi:10.1097/ MD.0000000000001439

11. Larroche C. Hemophagocytic lymphohistiocytosis in adults: diagnosis and treatment. Joint Bone Spine. 2012;79(4):356-361. doi:10. 1016/j.jbspin.2011.10.015

12. Duong TA. Infection due to Penicillium marneffei, an emerging pathogen: review of 155 reported cases. Clin Inf Dis. 1996;23 (1):125-130. doi:10.1093/clinids/23.1.125

13. Ma BH, Ng CS, Lam R, et al. Recurrent hemoptysis with Penicillium marneffei and Stenotrophomonas maltophilia in Job's syndrome. Can Respir J. 2016;16(4):e50-e52. doi:10.1155/2009/586919
14. Lee PP, Chan KW, Lee TL, et al. Penicilliosis in children without HIV infection-are they immunodeficient? Clin Infect Dis. 2012;54 (2):e8-e19. doi:10.1093/cid/cir754

15. Browne SK, Burbelo PD, Ploenchan C, et al. Adult-onset immunodeficiency in Thailand and Taiwan. N Engl J Med. 2012;367(8):725. doi:10.1056/NEJMoa1111160

16. Lee PPW, Mao H, Yang W, et al. Penicillium marneffei infection and impaired IFN- $\gamma$ immunity in humans with autosomal-dominant gainof-phosphorylation STAT1 mutations. J Allergy Clin Immunol. 2014;133(3):894-896.e895. doi:10.1016/j.jaci.2013.08.051

17. Egeler RM, Shapiro R, Loechelt B, Filipovich A. Characteristic immune abnormalities in hemophagocytic lymphohistiocytosis. J Pediatr Hematol Oncol. 1996;18(4):340-345. doi:10.1097/000 43426-199611000-00002

18. Tesi B, Sieni E, Neves C, et al. Hemophagocytic lymphohistiocytosis in 2 patients with underlying IFN-gamma receptor deficiency. $J$ Allergy Clin Immunol. 2015;135(6):1638-1641. doi:10.1016/j. jaci.2014.11.030

19. Chen J, Zhang R, Shen Y, et al. Clinical characteristics and prognosis of Penicilliosis among human immunodeficiency virus-infected patients in Eastern China. Am J Trop Med Hyg. 2017;96(6):13 50-1354. doi:10.4269/ajtmh.16-0521

20. Qiu Y, Liao H, Zhang J. Differences in clinical characteristics and prognosis of Penicilliosis among HIV-negative patients with or without underlying disease in Southern China: a retrospective study. $B M C$ Infect Dis. 2015;15(1):1-11. doi:10.1186/s12879-015-1243-y

21. Malinowska I, Machaczka M, Popko K, Siwicka A. Hemophagocytic syndrome in children and adults. Arch Immunol Ther Exp (Warsz). 2014;62(5):385-394. doi:10.1007/s00005-014-0274-1

22. Kashif M, Tariq H, Ijaz M, Gomez-Marquez J. Disseminated histoplasmosis and secondary hemophagocytic syndrome in a non-HIV patient. Case Rep Crit Care. 2015;2015:295735. doi:10.1155/2015/ 295735

23. Hu Y, Zhang J, Li X, et al. Penicillium marneffei infection: an emerging disease in mainland China. Mycopathologia. 2013;175(1-2):57-67. doi:10.1007/s11046-012-9577-0

24. Lei HL, Li LH, Chen WS, et al. Susceptibility profile of echinocandins, azoles and amphotericin B against yeast phase of Talaromyces marneffei isolated from HIV-infected patients in Guangdong, China. Eur J Clin Microbiol Infect Dis. 2018;37(6):1099-1102. doi:10.1007/ s10096-018-3222-x

25. Le T, Kinh NV, Cuc NTK, et al. A trial of itraconazole or amphotericin B for HIV-associated talaromycosis. N Engl J Med. 2017;376 (24):2329-2340. doi:10.1056/NEJMoa1613306
Infection and Drug Resistance

\section{Publish your work in this journal}

Infection and Drug Resistance is an international, peer-reviewed openaccess journal that focuses on the optimal treatment of infection (bacterial, fungal and viral) and the development and institution of preventive strategies to minimize the development and spread of resistance. The journal is specifically concerned with the epidemiology of antibiotic resistance and the mechanisms of resistance development and diffusion in both hospitals and the community. The manuscript management system is completely online and includes a very quick and fair peerreview system, which is all easy to use. Visit http://www.dovepress.com/ testimonials.php to read real quotes from published authors. 